\title{
CONTROLLER - PILOT COMMUNICATIONS USING A VDL MODE 2 DATALINK FOR THE NASA RUNWAY INCURSION PREVENTION SYSTEM ${ }^{1}$
}

\author{
Sanjeev Gunawardena \\ James M. Rankin \\ Avionics Engineering Center, Ohio University, Athens, Ohio
}

\begin{abstract}
NASA's Runway Incursion Prevention System (RIPS) was tested between September and October 2000 at the Dallas Fort-Worth International Airport (DFW). Controller - Pilot Datalink Communications (CPDLC) represented one component of the RIPS program. Air traffic controllers relayed surface operations instructions to NASA's 757 research aircraft via a Controller Communications and Situational Awareness Terminal (C-CAST). The Onyx mainframe computer aboard the 757 decoded these messages and sent automatic acknowledgements back to the C-CAST. The CPDLC messages were sent over a datalink utilizing the ICAO-defined VHF Digital Link (VDL) Mode 2 protocol. This paper presents implementation and performance details of the datalink. The paper describes the VDL Mode 2 protocol, the overall architecture of the CPDLC system, hardware-software interfacing details, CPDLC message formats, signal coverage data for the DFW airport, and design details of the Airborne Datalink Manager (ADLM) software used for this work.
\end{abstract}

\section{Introduction}

During 2000, the FAA and NASA cosponsored a program to develop a system to detect and avert runway incursions. The FAA program was called the Runway Incursion Reduction Program (RIRP) and the NASA program was called the Runway Incursion Prevention System (RIPS). Under the program, an experimental system using several emerging aeronautical technologies was installed at DFW. During the latter part of October
2000 , several flight tests were performed to test the system. The tests showed that the system was able to effectively warn pilots and controllers of runway incursions with adequate time to take corrective action and avoid impending disaster.

One goal of the system involved increasing situational awareness for both pilots and air traffic controllers (ATC). Developing an effective, efficient, and reliable communications system between controller and pilot was another. To achieve these goals, Ohio University's Avionics Engineering Center, in partnership with St. Cloud State University developed the C-CAST interface. C-CAST featured an active map display showing all airport traffic in real time. It also incorporated electronic flight strips to reduce the burden on the controller. The controller communicated with an aircraft by selecting it from the map display (by physically touching the item of traffic on the map) and speaking the ATC command using standard controller phraseology. C-CAST incorporated voice recognition technology to encode the controller's instruction into a standard ICAOdefined format. The textual instruction was displayed on the screen and transmitted to the aircraft after verification by the controller. Figure 1 shows the C-CAST active map display. Further details of C-CAST and its voice recognition technology can be found in [1] and [2] respectively.

\footnotetext{
${ }^{1}$ This research project was funded by the NASA-Langley Research Center, Hampton, VA.
}

\section{U.S. Government work not protected by U.S. Copyright.}




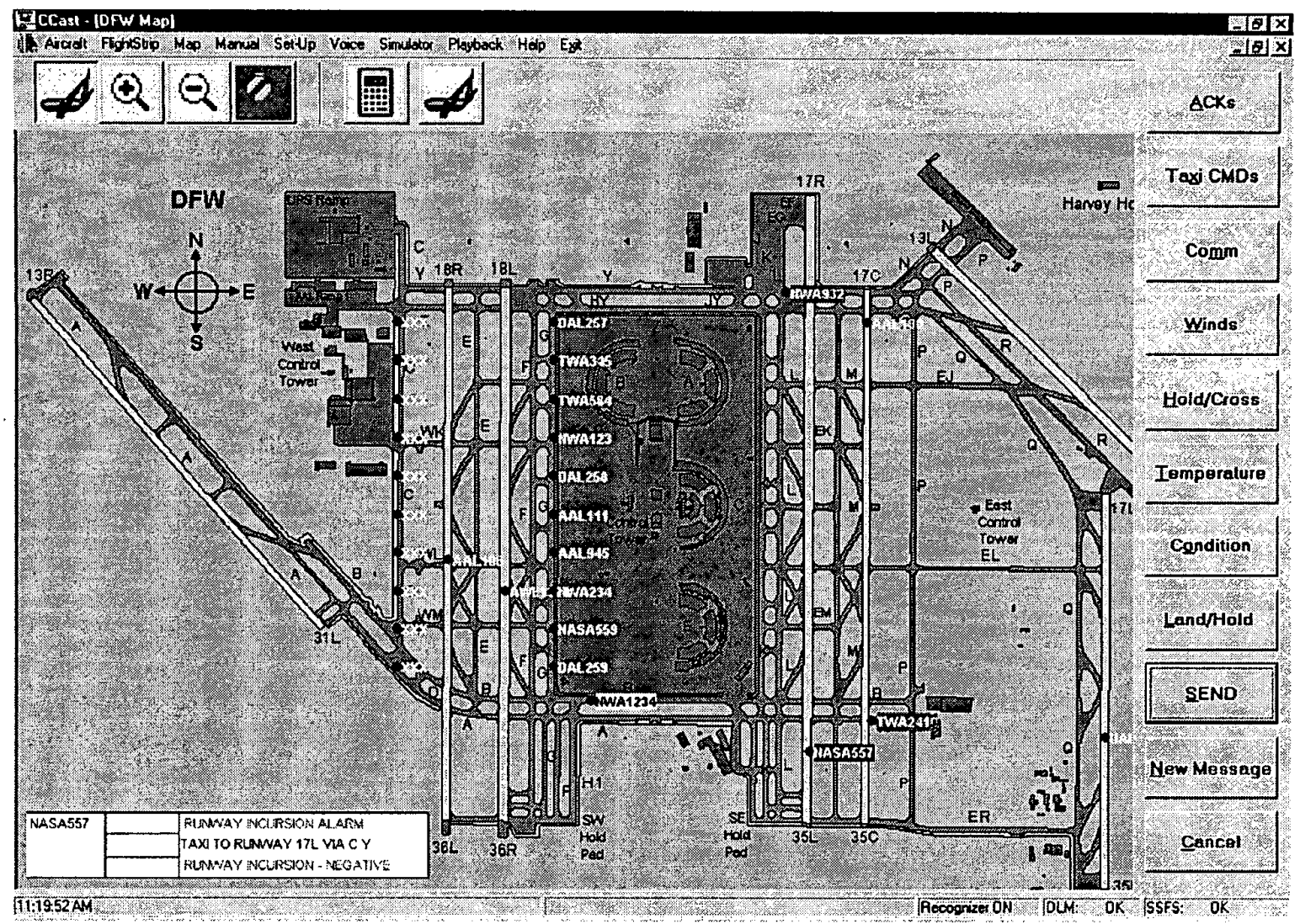

Figure 1. C-CAST Active Map Display

When the aircraft received the ATC instruction, its flight computer checked it for validity. If the message was understood, the airborne computer sent an acknowledgement to ATC. Surface operations instructions such as taxi route assignments were displayed on the pilot's electronic moving map (EMM), and caused appropriate guide symbols to appear on his headsup display (HUD). Other instructions such as "ALTIMETER" and "CONTACT TOWER" were displayed as text below the EMM. Figure 2 and Figure 3 show the EMM and HUD displays respectively, after the aircraft had received a "TAXI TO SPOT" instruction.

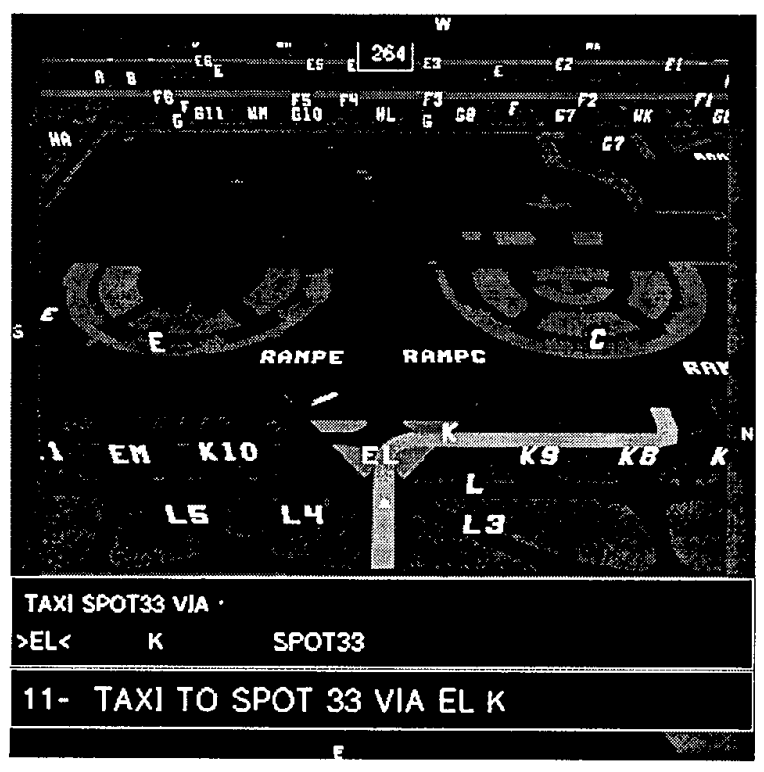

Figure 2. Electronic Moving Map Display 


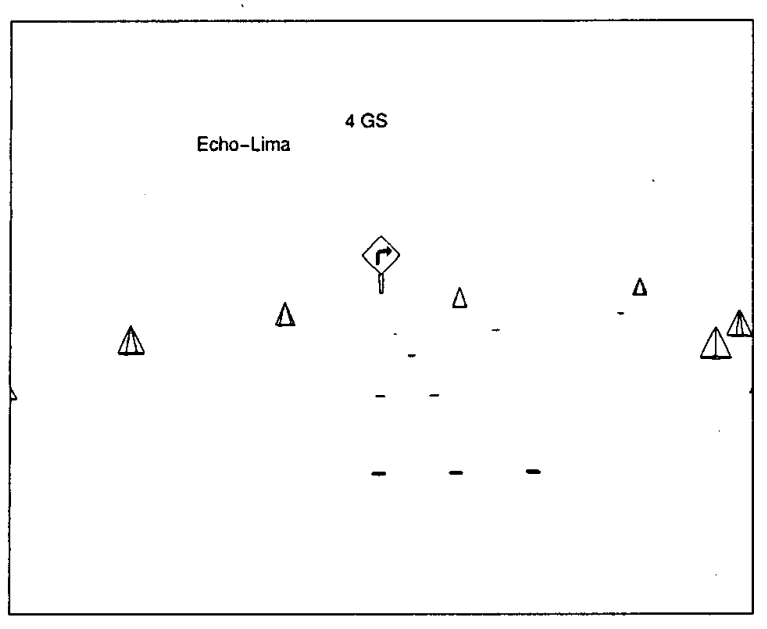

Figure 3. Pilot's Heads-Up Display

When acknowledgements were received by $\mathrm{C}$ CAST, the transmitted instructions displayed on the electronic flight strips changed color to indicate that the message was successfully received and understood by the aircraft. In addition to acknowledgements, the aircraft also sent other downlink messages such as status information and runway incursion alerts that provide valuable information to the controller.

The wireless datalink used to send and receive CPDLC messages was a crucial part of the RIPS. For example, if the airborne computer could not receive ATC taxi instructions, it was unable to display the taxi routes in the EMM and HUD, which could seriously impede the pilot's situational awareness capabilities, especially in low visibility conditions.

Figure 4 shows a high level diagram of the RIPS CPDLC subsystem. The C-CAST was physically located in the temporary NASA RIPS operational base located on the $15^{\text {th }}$ floor of the Harvey Hotel, which had a clear view of the East side runways where the runway incursion test scenarios were performed. The C-CAST was connected to the CPDLC Datalink Manager (CDLM) located at the East Control Tower via a wireless Ethernet connection. The CDLM and its airborne counterpart, the Airborne Datalink Manager (ADLM) handled interfacing to the radio equipment used to implement the datalink. As shown in Figure 4, the ADLM communicated with the airborne unit's Onyx mainframe computer via the standard TCP/IP networking protocol.

The CPDLC datalink for the DFW tests utilized the new aeronautical VHF digital data communications system defined by RTCA Special Committee 172 (SC-172). Specifically, the datalink used VDL Mode 2 defined in the standard. This paper describes the implementation and overall performance details of the RIPS CPDLC datalink.
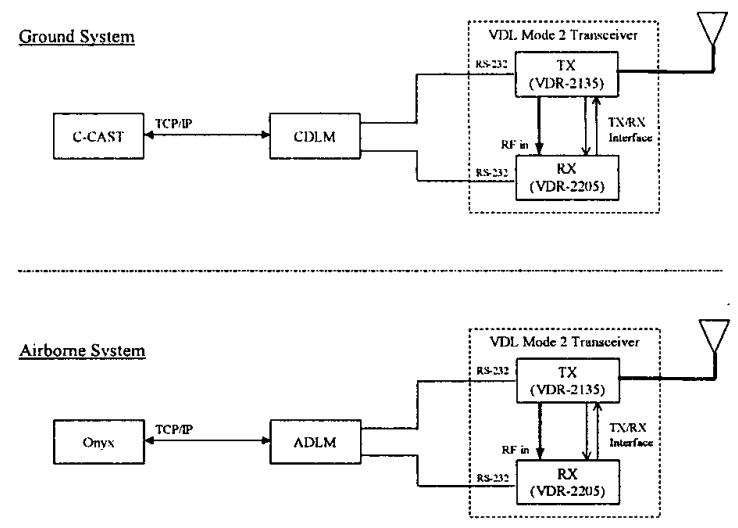

Figure 4. CPDLC Sub-System Architecture

\section{VDL Mode 2 Standard and Equipment}

As a replacement to the present doublesideband amplitude modulation (DSB-AM) based aeronautical VHF communications system operating within the radio frequency range $118.0-$ 137.0 MHz, SC-172 describes the Minimum Aviation System Performance Standards (MASPS) for an advanced VHF digital datalink communications system including capability for digital voice techniques [3]. Under this specification, two modes of operation are defined: VDL Mode 2 and VDL Mode 3.

VDL Mode 2 refers to the operation of a Carrier Sense Multiple Access (CSMA) scheme that supports datalink capabilities. CSMA allows multiple transmitters to share a single RF channel by having a connected receiver monitor the frequency for other transmissions. Once the channel is clear, a transmission is attempted based on a pre-configured set of statistical parameters. This statistical sharing of a channel results in efficient channel utilization. VDL Mode 2 is 
appropriate for aperiodic traffic where the entire message is ready before transmission of individual message packets is attempted. Due to the nature of CSMA, collisions are possible and hence this mode is not suited for time critical applications such as real time digital voice.

VDL Mode 3 refers to a functionally simultaneous voice and datalink capability using a Time Division Multiple Access (TDMA) architecture. Mode 3 is intended to provide a more deterministic service performance for time critical applications. Both modes use Differential EightPhase Shift Keying (D8PSK) modulation and support a nominal bit rate of $31.5 \mathrm{kbps}$.

Applications of the new air-ground communications system include Air Traffic Services (ATS), Aeronautical Operational Control (AOC) (first priority), and Aeronautical Administrative Communications (AAC) (lower priority). Since CPDLC messages are short, largely aperiodic messages belonging to the ATS category, a VDL Mode 2 implementation was chosen for the RIPS tests.

As defined in [3], the VDL Mode 2 Physical Layer provides the services to activate, maintain, and de-activate connections for bit transmission in the datalink layers. These services include receiver and transmitter frequency control, transmission and reception of data, and performance notification services.

The VDL Mode2 Link Layer is comprised of three sublayers: the Media Access Control (MAC) sublayer (which manages the CSMA algorithm), the Datalink Service (DLS) sublayer (to provide connection oriented point-to-point links using Data Link Entities (DLE) and a connectionless broadcast link over the MAC sublayer), and the VDL Management Entity (VME) sublayer (which establishes and maintains DLEs between airborne and ground stations).

The radio equipment used to implement the datalink consisted of a Harris VDR-2205 receiver and Harris VDR-2135 transmitter pair configured as a single transceiver. Both the air and ground stations used identical equipment except that the airborne units were flight-hardened by NASALangley. The VDR-2135 transmitters feature an internal solid-state transmit/receive antenna switch that greatly simplified the configuration of the units as transceivers.

The Harris radios implement all of the VDL Mode 2 Physical Layer and parts of its Link Layer. In particular, the radios implement the Link Layer's MAC sublayer and a portion of the DLS sublayer. The remaining portion of the DLS sublayer, the VME sublayer, and higher order application layers are implemented by the host computer's datalink manager software.

Interfacing to the radios was performed via their RS-232 data I/O port at $57.6 \mathrm{kbps}$. The datalink manager software supplied the Destination Address, Source Address, Control Byte, and the application (CPDLC) data components of an Aviation VHF Link Control (AVLC) frame [3]. The transmitter completed the frame by inserting the Start-of-Frame flag, the End-of-Frame Flag, the Frame Check Sequence, the Mode 2 Training Sequence, and the Forward Error Correction (FEC) field to complete the AVLC packet before transmission. Even though the transmitter has the capability to transmit several such AVLC frames in a single RF burst, only single-frame bursts were used for the RIPS tests.

Upon receipt of an RF burst, the receiver checks the FEC coding embedded in the message and corrects errors that may have occurred in the $\mathrm{RF}$ transmission. The receiver also performs the de-interleaving, de-scrambling, and removal of the header and training sequences before transferring the message to its host buffer, from where it will be passed to the datalink manager. Following the handoff of the AVLC frame to the host, the receiver reports the following parameters pertaining to the received RF burst: signal quality, received signal strength (RSS), number of symbols received, number of bytes and blocks corrected with FEC algorithm, confidence factor, end of burst indication and a broken message indication. These parameters were logged by the datalink manager for subsequent datalink performance analysis.

\section{VDL Mode 2 Coverage Testing at DFW}

To ensure that the VDL-Mode 2 datalink will work reliably for the RIPS CPDLC operations, coverage tests were performed at DFW during 
August 2000. A temporary ground station was setup as described in the Ground Station section of this paper. A van was used to simulate an aircraft on the surface of the airport. The airborne transceiver was installed in the van. The van antenna was a standard $1 / 4$ wave whip antenna placed on its roof using a magnetic mount. The datalink manager software that controlled the airborne transceiver was configured to transmit a CPDLC downlink "ROGER" message approximately every second. The ground station datalink manager was configured to reply to this message with the corresponding uplink "ROGER" message. The transmitted and received messages at both ends, along with their reported VDL Mode 2 parameters were time stamped and logged for analysis. The tests were performed on two consecutive nights when airport traffic was minimal. During the tests, the van traveled along most runways and taxiways on the East side of the airport, and some West side taxiways that were to be used for the RIPS flight tests. Airport surface coverage plots were generated by correlating the received messages' time stamps with GPS position and plotting that location's received signal strength. The coverage from ground-to-van (uplink) and vanto-ground (downlink) for both days of testing are shown in Figures 5 thru 8.

As evident in the plots, more coverage data was collected on the second day of testing. Comparisons of the plots show that the results obtained were roughly the same for both days of testing. Comparison between the uplink versus downlink plots show that the downlink plots have slightly better received signal strength. The reason for this being that the ground antenna was a narrowband type tuned exactly to the frequency of operation, hence providing higher gain. Careful observation of the plots reveals areas of severe signal attenuation due to obstructions. For example, the signal received at the mid-section of runway $31 \mathrm{R}$ and the corresponding $\mathrm{Q}$ and $\mathrm{R}$ taxiways is attenuated due to obstruction by the East control tower. Similar attenuation occurs on runway $35 \mathrm{R}$ due to obstruction by the Delta Airways hangar. In this respect, the terminal buildings on the center of the airport are the most significant obstructions since they block the signal path for much of the West side of the airport. Even though the received signal strength at the West side was frequently less than $-90 \mathrm{dBm}$, no messages had bit errors and there were no missed messages detected for both days of testing. This proved the reliability and robustness of the VDL Mode 2 datalink and provided a high degree of confidence that the datalink would work well during the RIPS flight tests.

\section{CPDLC Message Structure}

Selected messages from the ATN SARPS [4] and RTCA DO-219 [5] plus new messages for the airport surface environment were implemented in the C-CAST. The CPDLC message set implemented for the RIPS is defined in [6]. Following is a summary of these uplink and downlink messages:

Uplink Messages

- UNABLE $^{2}$

- STANDBY $^{2}$

- REQUEST DEFERRED ${ }^{2}$

- ROGER $^{2}$

- CONTACT [IcaoUnitName] [Frequency] ${ }^{2}$

- MONITOR [IcaoUnitName] [Frequency] ${ }^{2}$

- ALTIMETER [Altimeter] ${ }^{2}$

- [Freetext]

- HOLD SHORT OF [Position]

- TAXI RUNWAY [Runway] VIA [TaxiRoute]

- TAXI RAMP [Ramp] VIA [TaxiRoute]

- CROSS [Position] [WITHOUT DELAY]

- CONTINUE TAXI

- UNAVAILABLE TAXIWAYS [Taxiways]

- RUNWAY [Runway] TAXI INTO POSITION AND HOLD

- RUNWAY [Runway] CLEARED FOR TAKEOFF

- WIND [Direction] AT [Speed]

- RUNWAY CONDITION [Condition]

- LAND AND HOLD SHORT OF [Runway]

- TAXI TO GATE [GateNumber] VIA [TaxiRoute]

- TEMPERATURE [TemperatureC]

- RUNWAY [Runway] CLEARED to LAND

- TAXI TO SPOT [SpotNumber] VIA [TaxiRoute] 


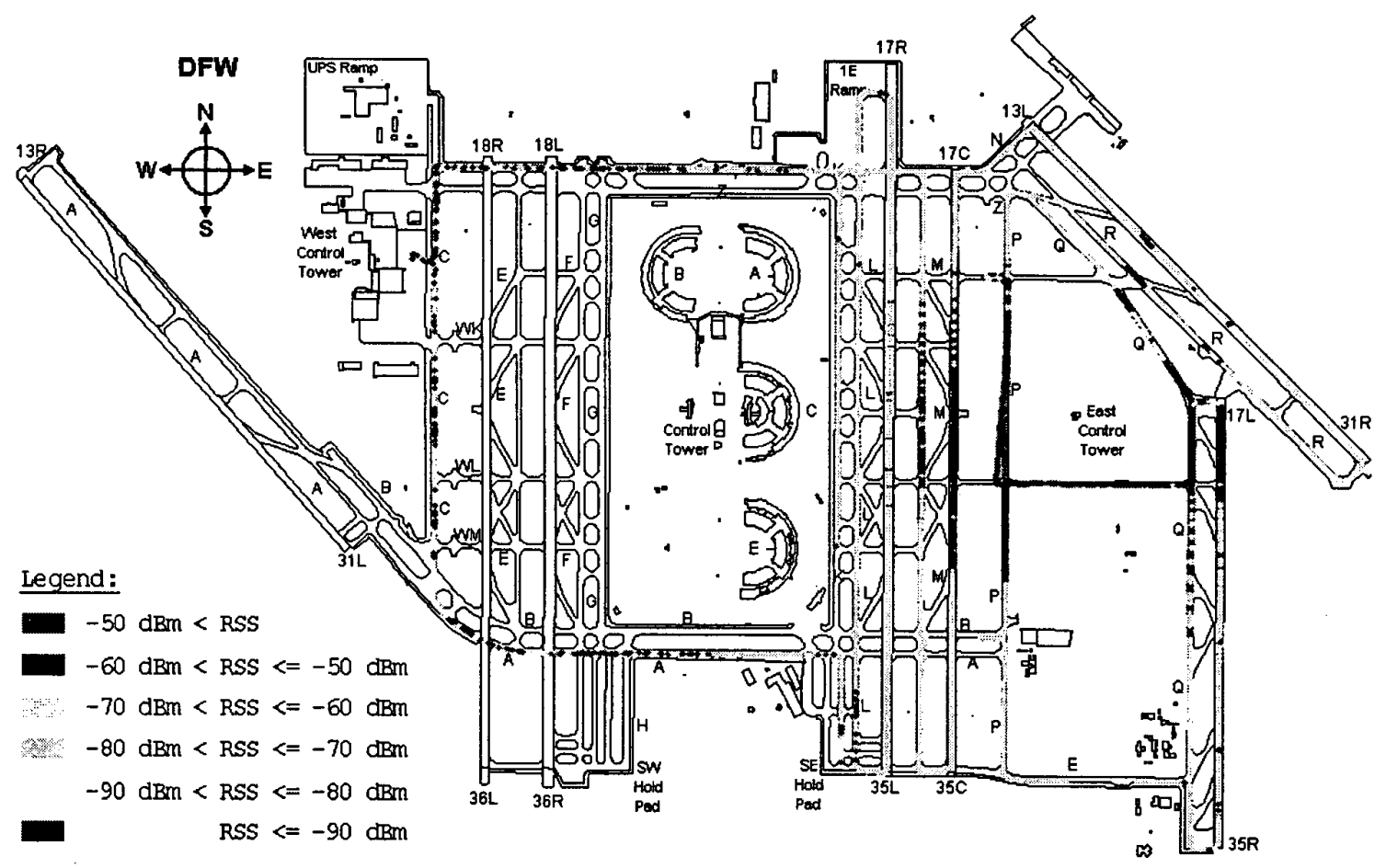

Figure 5. Ground-to-Van (Uplink) Coverage 8-23-00

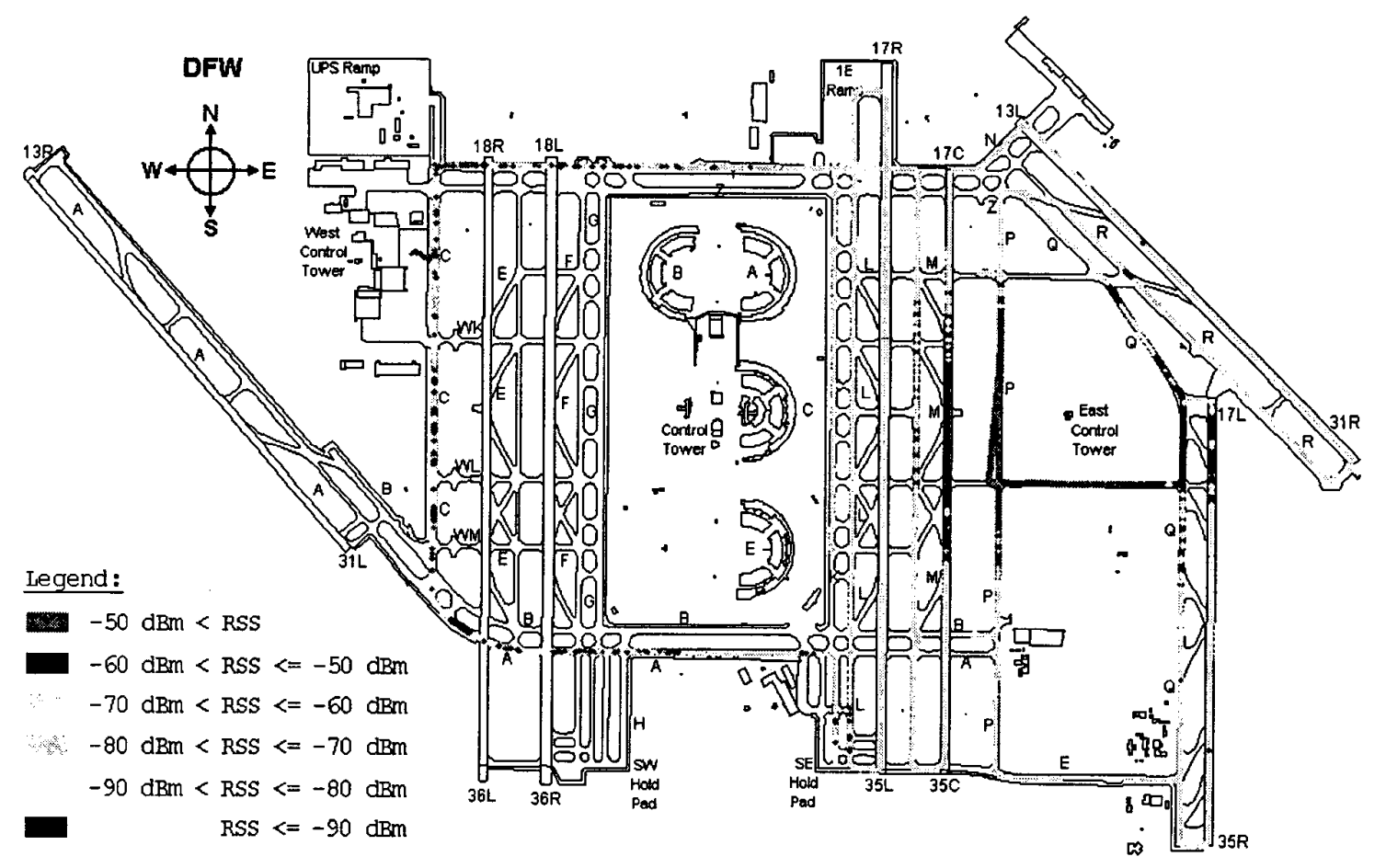

Figure 6. Van-to-Ground (Downlink) Coverage 8-23-00 


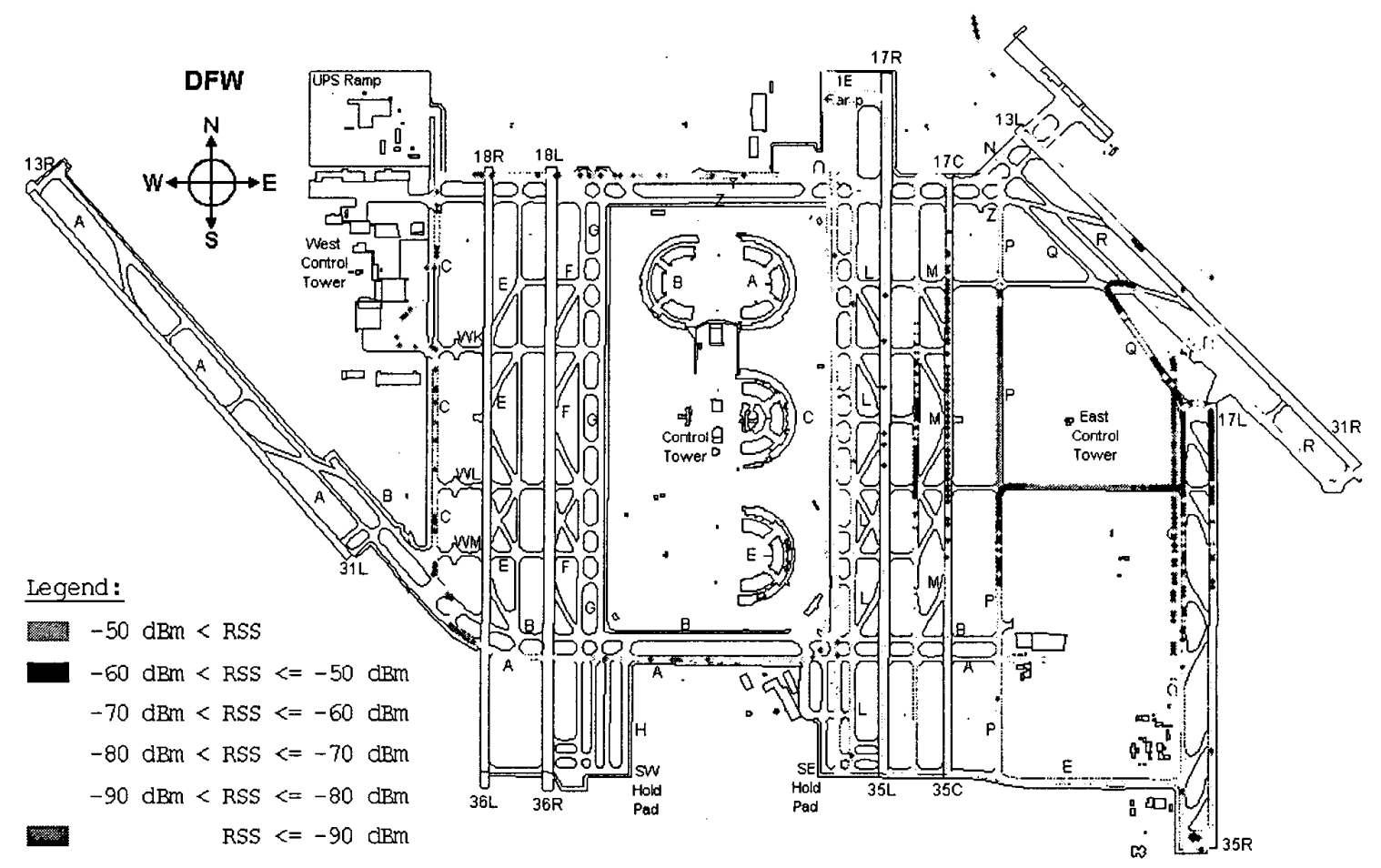

Figure 7. Ground-to-Van (Uplink) Coverage 8-24-00

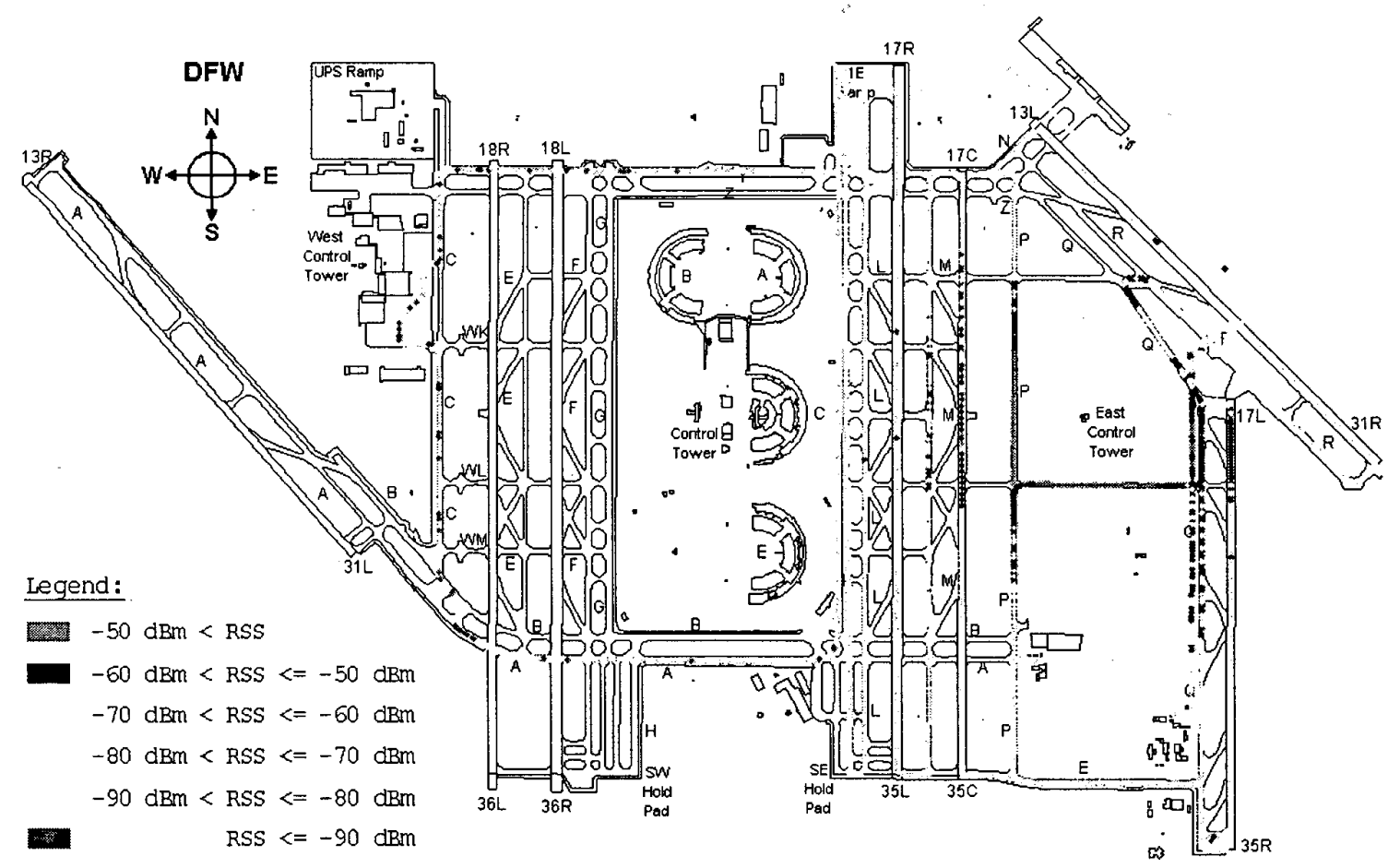

Figure 8. Van-to-Ground (Downlink) Coverage 8-24-00 
Downlink Messages

- $\mathrm{UNABLE}^{2}$

- ROGER $^{2}$

- LANDING REPORT

- REQUEST TAXI CLEARANCE

- TAXI DEVIATION

- TURNED-OFF ON TAXIWAY [Taxiway]

- TAXI DEVIATION RESOLVED

- RUNWAY INCURSION [Source] [AlarmType] [Identification]

- ASSIGNED GATE [GateNumber]

These messages and their associated parameters were encoded into a variable length packet according to the encoding rules defined in [4]. The encoding rules are designed to minimize the overall length of the packet. CPDLC packet encoding details can be found in [6].

The CPDLC data packet was then embedded into a CPDLC message structure before being sent out to the CDLM or ADLM via TCP/IP. The format of this structure was largely influenced by the requirements of the VDL AVLC frame specification. Table 1 presents the CPDLC message structure.

The Message Type byte determined how the message was handled by the datalink nodes. In general, the Message Type range $0 \times 10$ to $0 \times 1 F$ was reserved for uplink messages (ground-to-air) and the range $0 \times 20$ to $0 \times 2 \mathrm{~F}$ was reserved for downlink (air-to-ground) messages. All message type definitions used for the RIPS tests are given in Table 2.

Since the CPDLC message structure had variable length, a Message Length field was needed so that the parsing software could properly identify the end of the message. The destination and source address fields contained the 23-bit ICAO addresses encoded according to [4]. The Control Byte was included for AVLC frame completeness but was not used for the RIPS tests.

\footnotetext{
${ }^{2}$ Defined in ICAO ATN Standards and Recommended Procedures (SARPS) Manual [4]
}

Table 1. General CPDLC Message Structure

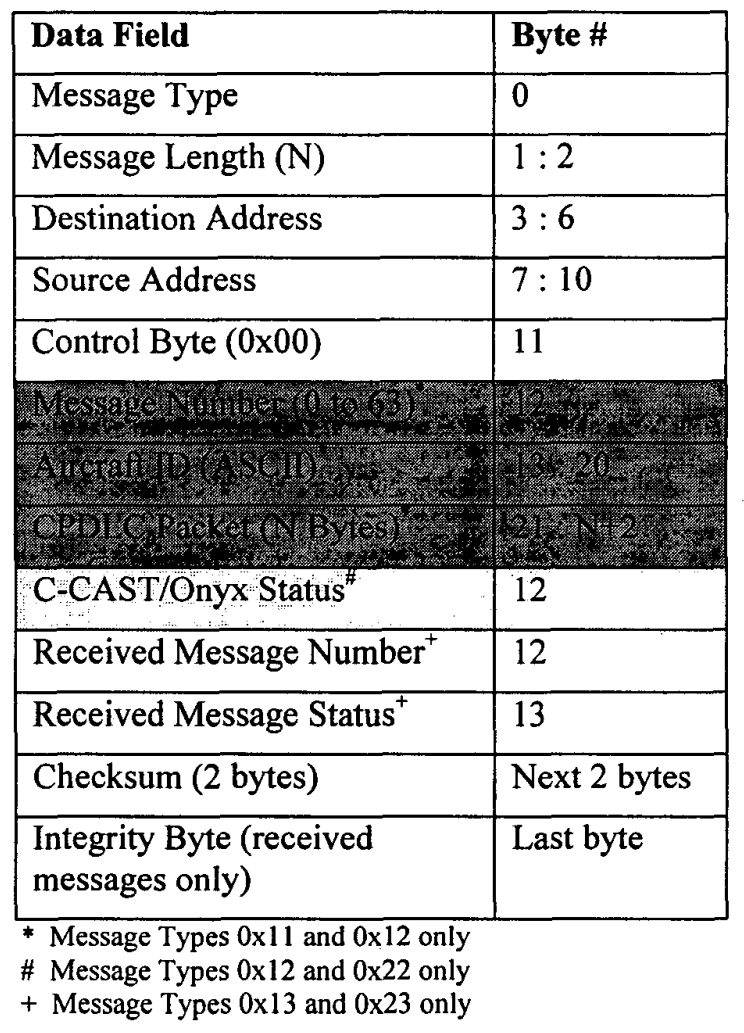

Table 2. CPDLC Message Type Definition

\begin{tabular}{|l|l|}
\hline Value & Message Type Definition \\
\hline $0 \times 11$ & CPDLC Uplink Message \\
\hline $0 \times 12$ & C-CAST Status \\
\hline $0 \times 13$ & $\begin{array}{l}\text { C-CAST Downlink Status } \\
\text { (CPDLC Downlink Acknowledge) }\end{array}$ \\
\hline $0 \times 21$ & CPDLC Downlink Message \\
\hline $0 \times 22$ & Onyx Status \\
\hline $0 \times 23$ & $\begin{array}{l}\text { Onyx Uplink Status } \\
\text { (CPDLC Uplink Acknowledge) }\end{array}$ \\
\hline
\end{tabular}

A CPDLC uplink or downlink message contained three other information fields; Message Number, an ASCII Aircraft ID, and the CPDLC data packet described previously. The Message Number uniquely identified the CPDLC uplink or downlink on a short-term basis for 
acknowledgement purposes. The Aircraft ID field, though filled with data, did not serve a useful purpose for the RIPS tests.

In addition to the main uplink and downlink messages that carry the CPDLC data packets, two other message categories were defined: Status Messages and Acknowledgement Messages. Status Messages were continuously transmitted at 15 Second intervals by both the C-CAST and Onyx mainframe. These transmissions indicated that the ground or airborne components were operational. They also provided a method to log data at constant intervals to analyze the performance of the datalink. In a practical sense, the Onyx status messages enabled C-CAST to track the aircraft in a positionless "CPDLC-based tracking" mode and send taxi instructions when the aircraft was on the West end of DFW (American Airlines hanger) where the RIPS traffic surveillance system did not operate.

Acknowledgement Messages were sent immediately following the receipt of a valid CPDLC packet. The Acknowledgement Message referred to the received CPDLC message's Message Number along with a Received Message Status byte. The Received Message Status byte would be non-zero if the receiver had reported low confidence for the received message, which represented a request to re-transmit that message. C-CAST, upon receipt of an acknowledgement with a Received Message Status value of zero, changed the color of the corresponding ATC instruction on the flight strip to indicate to the controller that the instruction was properly received by the aircraft.

A two-byte Checksum field was added to each CPDLC message before being sent out to any node for data integrity checking purposes. For all messages received by a VDL Mode 2 receiver, an Integrity Byte was attached to the end of the CPDLC message packet. The Integrity Byte was a summary of the VDL Mode 2 received burst quality reporting parameters generated by the receiver. The datalink manager software set the corresponding bits of the Integrity Byte when the parameters exceeded a preset threshold. The Integrity Byte definition is given in Table 3 .

Further details of the CPDLC message structure and hardware-software interfacing issues relating to the RIPS tests can be found in [7].
Table 3. Integrity Byte Definition

\begin{tabular}{|l|l|}
\hline Bit \# & Message Type Definition \\
\hline $7(\mathrm{msb})$ & Broken message \\
\hline 6 & FEC algorithm failure \\
\hline 5 & Confidence factor threshold not met \\
\hline 4 & Not used $(0)$ \\
\hline 3 & Not used $(0)$ \\
\hline 2 & Not used $(0)$ \\
\hline 1 & Signal quality threshold not met \\
\hline $0(\mathrm{lsb})$ & Signal strength threshold not met \\
\hline
\end{tabular}

\section{Ground Station}

The ground station for the CPDLC datalink was located within the East Tower compound. Figure 9 shows the radio equipment and host computer that was located inside the temporary RIRP trailer.

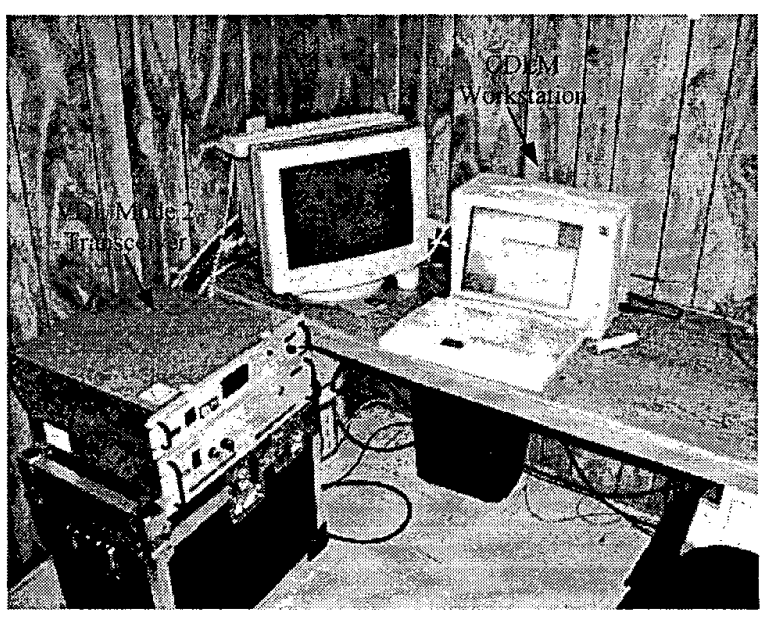

Figure 9. CPDLC Ground Station Equipment

A standard, vertically polarized, VHF aeronautical communications antenna tuned to the assigned frequency was used for the ground station. The antenna was erected along the West side wire fence of the East Tower compound. Antenna height was approximately 16 feet above ground level. Figure 10 shows the CPDLC ground station antenna. The RIRP trailer is visible in the background. 


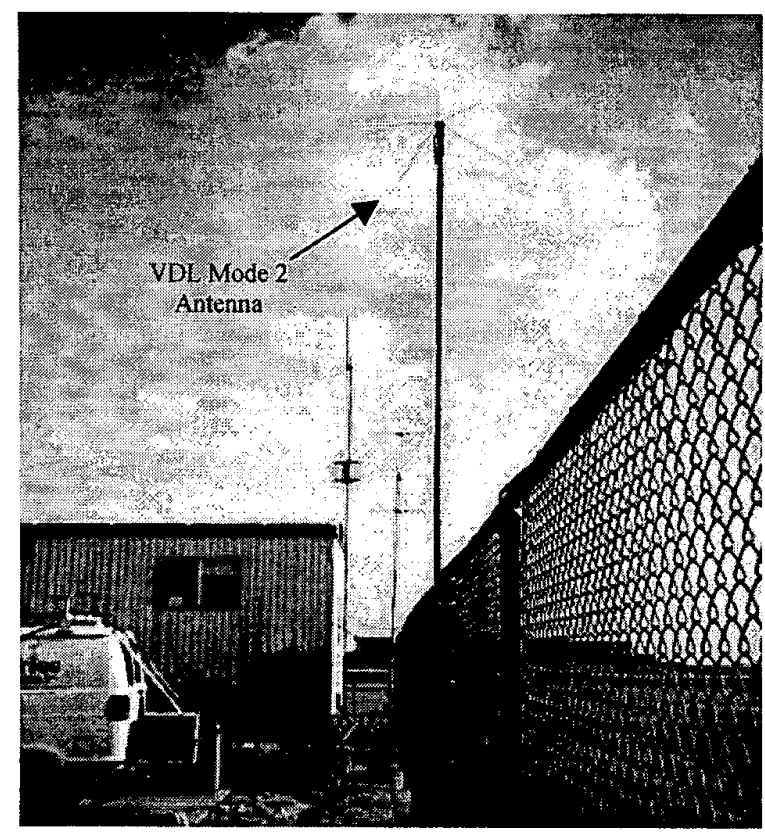

Figure 10. CPDLC Ground Station Antenna

\section{Airborne Unit}

As mentioned previously, the airborne radio equipment was identical to that of the ground station. The radios were installed in the Technology Transfer Area 2 (TTA2) equipment palette situated near the aft of NASA's Boeing 757 Airborne Research and Integrated Experimental System (ARIES) aircraft. ARIES contains numerous equipment palettes; each equipped with standard line power to enable non-airborne equipment to be easily adapted for flight tests. A $300 \mathrm{MHz}$ Pentium II ${ }^{\mathrm{TM}}$ based FieldWorks ${ }^{\mathrm{TM}}$ workstation running the Windows-NT ${ }^{\mathrm{TM}}$ operating system hosted the Airborne Datalink Manager (ADLM). Figure 11 shows TTA2 and the ADLM host computer. The airborne antenna was a standard blade-type wide-band VHF aeronautical communications antenna. Figure 12 shows the antenna's location on ARIES. The ADLM connected to the Onyx mainframe via standard Ethernet cabling, which in turn routes to a fiberoptic $1 / O$ ring known as the ScramNet. For time stamping purposes, the ADLM workstation was time synchronized using the aircraft's IRIG-B network.

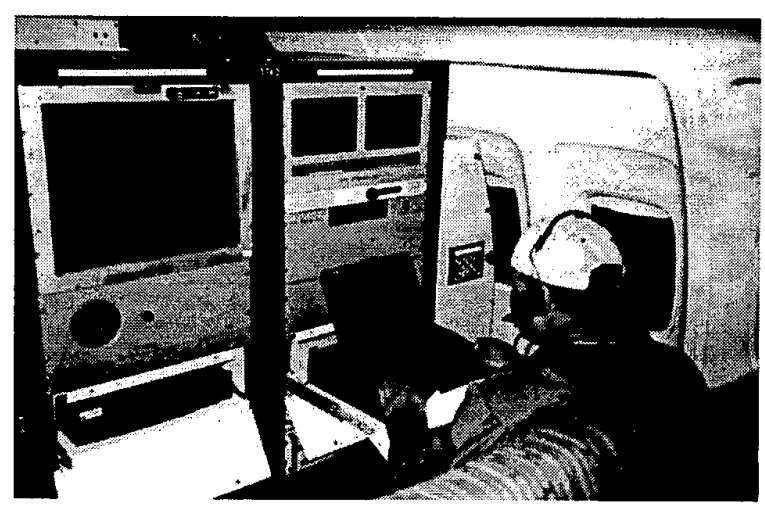

Figure 11. TTA2 Showing ADLM Workstation

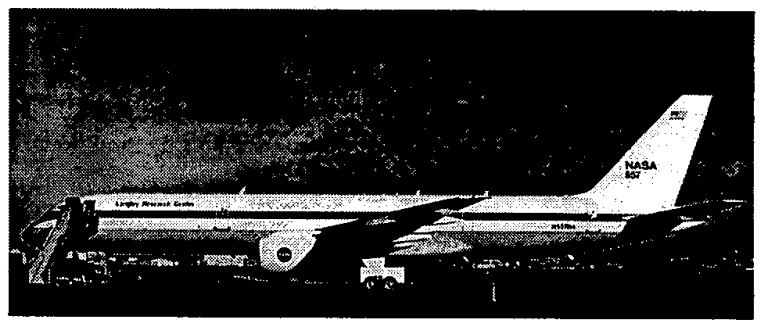

Figure 12. ARIES Showing VDL Mode 2 Antenna

\section{CDLM/ADLM Design Details}

The CDLM and ADLM software performed the interfacing between the Harris VDL Mode 2 radios and the C-CAST and Onyx mainframe respectively. Originally the CDLM and ADLM were to be developed as two separate entities. However since their functionality was similar, the software was written such that it could be configured to behave as either the CDLM or the ADLM. Hence, the following description of the ADLM also applies to the CDLM.

The ADLM was written in $\mathrm{C}++$ using the Borland $\mathrm{C}++$ Builder software development environment. Borland's Rapid Application Development (RAD) technology enabled the ADLM to be developed easily within a limited time frame. Functional blocks within the ADLM were developed as software objects that facilitated these objects to be reused. One of the main functions of the ADLM was to interface with the Harris radios using their binary RS-232 data $\mathrm{I} / \mathrm{O}$ ports. The complete RS-232 protocol to interface to the radios and all handshaking and error detection functions 
were integrated into a single software object. This object was written so that it could be configured to behave as the interface for either the receiver or the transmitter. Hence the ADLM used two instantiations of this object to interface to the combined transceiver. The ADLM used the TCP/IP client socket objects available in the Borland development suite to implement the Ethernet client socket. When a message was received from the Onyx, the ADLM first performed a checksum validation to determine that the message was received correctly. It then parsed the Message Type byte to determine what operation was to be performed with the message. If the message was meant for transmission, the ADLM re-arranged the message into the format required by the transmitter and invoked the transmitter's Fill Buffer command, which sends the data packet to the transmitter. Once a message is transmitted, the transmitter sends an acknowledgement that the message (with a given reference number) was transmitted successfully. The ADLM checked for this acknowledgement to verify that the message was successfully sent.

When a message was received by the receiver, it sent the received AVLC packet to the ADLM. The ADLM verified that the message was valid through its checksum. The ADLM also performed various ambiguity resolution routines to extract the AVLC frame. The AVLC frame was then packaged into the CPDLC message format given in Table 1. Upon receiving a message, the receiver outputs various VDL Mode 2 parameters pertaining to the quality of the received RF burst. The ADLM compared these parameters to preset thresholds and attached the resulting Integrity Byte (Table 3 ) to the end of the CPDLC message before it was sent to the Onyx.

In addition to the basic error checking, repackaging, and relaying of CPDLC messages between the Onyx and the VDL Mode 2 transceiver, the ADLM performed configuration functions such as setting frequency, output power, VDL Mode 2 link parameters, etc. for the radios. The ADLM also time stamped and logged all received and transmitted messages, all VDL Mode 2 parameters reported by the receiver, and any other messages reported by the radios for data analysis.

For datalink debugging purposes the ADLM incorporated several test modes to independently check each applicable part of the CPDLC datalink. These included automatic generation and transmission of CPDLC "ROGER" downlink messages at a configurable interval, automatic acknowledgement of a CPDLC uplink message by transmitting the corresponding downlink "ROGER" message, and modes to perform the equivalent functions to test the TCP/IP link.

The ADLM's Graphical User Interface (GUI) was divided into four main panels, as shown in Figure 13. These allowed a user to see at a glance, the operational mode of the ADLM and the state of the receiver, transmitter, and TCP/IP link in real time. Activity indicators displayed RS-232 and $\mathrm{TCP} / \mathrm{IP}$ traffic between the transmitter, receiver, and Ethernet link by changing color (between yellow and green) whenever messages were sent or received by these components. The tab sheet at the bottom of the GUI allowed the user to monitor the CPDLC messages flowing through the ADLM in either binary, transcript, decoded CPDLC, or log file formats. These features helped to debug numerous problems during the many RIPS system checkout phases and enabled the user to verify that the CPDLC datalink was operating properly during all flight tests.

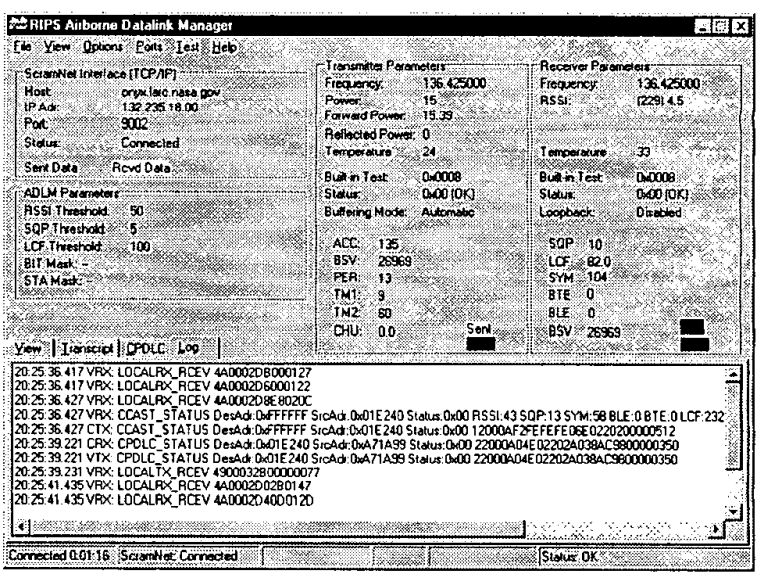

Figure 13. CDLM/ADLM User Interface

\section{Conclusions}

Consistent with the conclusions drawn from the coverage tests, the datalink worked flawlessly during all five data collection, and two system demonstration flights. During all flight tests, both 
the CDLM and ADLM consoles were monitored for proper operation of the radios, the software, and the datalink in general. No critical failure of either the radios or the software was detected. All CPDLC uplink, downlink, and acknowledgement messages were sent and received correctly as best monitored through the consoles. Attempts are underway to analyze all data collected during the flight tests. Preliminary results show that a total of 1021 CPDLC uplink and downlink messages were transmitted for all five data collection flights. Of these, the analysis shows a total of 5 messages failed to be received by either the C-CAST or the Onyx. Hence the datalink had an overall reliability of $99.51 \%$. Conclusive analysis is underway to determine which section of the datalink was responsible for these dropped messages. Overall, the VDL Mode 2 datalink described in this paper performed above expectations to support CPDLC operations for all RIPS flight tests performed at DFW.

\section{Summary}

This paper described the implementation of a VDL Mode 2 datalink to support CPDLC operations for the NASA RIPS flight tests at DFW. The datalink established controller-pilot datalink communications between the ATC C-CAST interface and the NASA-Langley 757 research aircraft. The datalink was implemented using third party radio equipment controlled by datalink manager software developed by the Ohio University Avionics Engineering Center. Qualitatively, the datalink worked flawlessly during the data collection and system demonstration flight tests. Preliminary analysis of the data collected during these flights support this claim.

\section{Acknowledgements}

The authors wish to thank Denise Jones, David Green, Charles Howell, Brian Hutchinson, and Sharon Otero of the NASA-Langley Research Center and Vernon Edwards of the FAA for assistance provided prior to and during the RIPS flight tests. NASA-Langley's Paul Hyer is thanked for generating the EMM and HUD graphics used in this paper. Ohio University Avionics Engineering Center personnel, namely, Eric Best, Michael DiBenedetto, Carl Hawes, Qingwei Ma, and Rob
Thomas provided immense support and assistance during the coverage and flight tests. AEC's Frank Alder is thanked for providing preliminary data analysis results.

\section{References}

[1] Best, Eric, J. Rankin, Oct. 2001, ControllerPilot Data Link Results from NASA and FAA Dallas-Fort Worth 2001 Test and Demonstration, Proceedings of the $20^{\text {th }}$ DASC.

[2] Lechner, Alicia, K. Ecker, P. Mattson, Oct. 2001, Voice Recognition - Software Solutions in Real Time ATC Workstations, Proceedings of the $20^{\text {th }}$ DASC.

[3] Sept. 2000, DO-224A Signal-in-Space Minimum Aviation System Performance Standards (MASPS) for Advanced VHF Data Communications Including Compatibility with Digital Voice Techniques, RTCA Inc.

[4] 1999, Manual of Technical Provisions for the Aeronautical Telecommunications Network (ATN), $2^{\text {nd }}$ edition, International Civil Aviation Organization.

[5] Aug. 1993, DO-219 Minimum Operational Performance Standards for ATC Two-Way Data Link Communications, RTCA Inc.

[6] Rankin, James, Jul. 2000, C-CAST Message Set for DFW RIPS Demonstration, Avionics

Engineering Center, Ohio University.

[7] Gunawardena, Sanjeev, J. Rankin, Jul. 2000, Airborne Datalink Manager to NASA-Langley B757 ScramNet Interface Control Document, Avionics Engineering Center, Ohio University.

[8] Young, Steve, J. Rankin, Nov. 1998, Improving the Safety of Airport Surface Operations, Air Traffic Technology International.

[9] Rankin, James, P. Mattson, 1998, ControllerPilot Data Link Statistics from NASA's 1997 Atlanta Flight Test, Proceedings of the $17^{\text {th }}$ DASC.

[10] Rankin, James, P. Mattson, 1997, Controller Interface for Controller-Pilot Data Link Communications, Proceedings of the $16^{\text {th }}$ DASC. 